OPEN ACCESS

Edited by:

Rocío Martínez De Pablos,

University of Seville, Spain

Reviewed by:

Shafiq Ur. Rehman,

Gyeongsang National University,

South Korea

Antonio Boza Serrano,

Lund University, Sweden

*Correspondence:

Jiachun Feng

fjcfrank@qq.com

Mingqin Zhu

mingqin.zhu@hotmail.com

${ }^{t}$ These authors have contributed equally to this work

Specialty section:

This article was submitted to

Cellular Neuropathology,

a section of the journal

Frontiers in Cellular Neuroscience

Received: 09 May 2019

Accepted: 30 September 2019

Published: 15 October 2019

Citation:

Yin P, Wang $X$, Wang S, Wei Y,

Feng $J$ and Zhu $M$ (2019) Maresin 1

Improves Cognitive Decline

and Ameliorates Inflammation in a

Mouse Model of Alzheimer's Disease.

Front. Cell. Neurosci. 13:466.

doi: 10.3389/fncel.2019.00466

\section{Maresin 1 Improves Cognitive Decline and Ameliorates Inflammation in a Mouse Model of Alzheimer's Disease}

\author{
Ping Yin ${ }^{1,2 t}$, Xu Wang ${ }^{1+}$, Shuang Wang ${ }^{1}$, Yafen Wei ${ }^{1,2}$, Jiachun Feng ${ }^{1 *}$ and Mingqin Zhu ${ }^{1 *}$ \\ ${ }^{1}$ Department of Neurology and Neuroscience Center, The First Hospital of Jilin University, Changchun, China, ${ }^{2}$ Department \\ of Neurology, Heilongjiang Provincial Hospital, Harbin, China
}

Alzheimer's disease (AD) is one of the most common neurodegenerative disease. Accumulating evidences suggest an active role of inflammation in the pathogenesis of AD. Inflammation resolution is an active process that terminates inflammation and facilitates the restoration of inflamed tissue to homeostasis. Resolution of inflammation has been shown to be conducted by a group of specialized pro-resolving lipid mediators (SPMs) including lipoxins, resolvins, protectins, and maresins (MaRs). Recent studies have demonstrated that failure of inflammation resolution can lead to chronic inflammation and, hence, contribute to $A D$ progression. We have previously shown that MaR1 can improve neuronal survival and increase microglial phagocytosis of $A \beta$. However, the effects of MaR1 on animal models of AD have not been reported. In this study, we aim to investigate the effects of MaR1 on behavioral deficits and pathological changes in a mouse model of $A D$. Mice received bilateral injections of $A \beta_{42}$ protein into the hippocampus, followed by administration of MaR1 by intra-cerebroventricular injection. The behavioral changes in the mice were analyzed using Morris water maze. Immunohistochemistry, Fluoro-Jade B (FJB) staining, cytometric beads array (CBA), and western blot analysis were used to demonstrate molecular changes in the mice hippocampus and cortex. Our results showed that MaR1 treatment significantly improved the cognitive decline, attenuated microglia and astrocyte activation. In addition, we found that MaR1 decreased the pro-inflammatory cytokines TNF- $\alpha, I L-6$, and MCP-1 production induced by $\mathrm{A} \beta 42$ and increased the anti-inflammatory cytokines IL-2, IL-10 secretion with or without A $\beta 42$ stimulation. Moreover, western blot results showed that MaR1 up-regulated the levels of proteins related to survival pathway including PI3K/AKT, ERK and down-regulated the levels of proteins associated with inflammation, autophagy, and apoptosis pathways such as p38, mTOR and caspase 3. To conclude, MaR1 improved the cognitive decline, ameliorated pro-inflammatory glia cells activation via improving survival, enhancing autophagy, inhibiting inflammation and apoptosis pathways. In conclusion, this study shows that inflammation resolution may be a potential therapeutic target for AD.

Keywords: Alzheimer's disease, resolution of inflammation, maresin 1, neuroinflammation, memory 


\section{INTRODUCTION}

Alzheimer's disease (AD) is the most common cause of dementia, with no existing treatment that can significantly delay or reverse $\mathrm{AD}$-associated cognitive decline or pathological changes. The gross histopathology of $\mathrm{AD}$ is characterized by brain atrophy, deepening of the cerebral grooves and the enlargement of the cerebral ventricles. Histologically, $A D$ is characterized by extracellular deposition of amyloid- $\beta$ ( $A \beta)$, intracellular accumulation of neurofibrillary tangles (NFTs) (Alzheimer et al., 1995), and chronic inflammation (McGeer et al., 1987, 1988; Griffin et al., 1989; Cacabelos et al., 1994). A $\beta$ has been considered as a main culprit of $\mathrm{AD}$, and the $\mathrm{A} \beta$ hypothesis has been dominant for explaining the pathogenesis of AD (Hardy, 2009). There is a plethora of evidence that support the $A \beta$ hypothesis: patients with an extra copy of chromosome 21, where the APP gene locates, develop dementia at an early age; APP transgenic mice exhibit significant cognitive impairment; the toxicity of $A \beta$ has been documented extensively in in vitro studies (Del Bo et al., 1995; Combs et al., 2001; Wicklund et al., 2010). Results from genetic studies have shown an association of inflammation-related genes with $\mathrm{AD}$ (Griciuc et al., 2013). Further, microglia activation as well as elevated proinflammatory mediators observed in postmortem $\mathrm{AD}$ brains and in $\mathrm{AD}$ mice models support that chronic inflammation is an integral part of $\mathrm{AD}$ pathogenesis (Heppner et al., 2015). Inflammation resolution is an active regulatory process in the end stage of inflammatory reaction that can terminate inflammation and initiate repair of damaged tissues rather than passive disappearance of inflammatory mediators as previously believed (Serhan, 2017). Inflammation resolution is mediated by a group of lipid mediators called specialized pro-resolving lipid mediators (SPMs) including lipoxins (LXs), resolvins (Rvs), protectins (NPDs), and maresins (MaRs), all of which are biosynthesized from polyunsaturated fatty acids (PUFAs) via cyclooxygenases (COXs) and lipoxygenases (LOXs) (Serhan, 2014). In humans, studies have found that reduced SPMs lead to failure of inflammation resolution that can contribute to chronic inflammation diseases such as atherosclerosis (Fredman et al., 2016), dry eye pathogenesis (Gao et al., 2015) as well as AD (Wang et al., 2015).

Evidences from recent studies demonstrate that inflammation resolution is impaired in $\mathrm{AD}$ and stimulation of inflammation resolution showed beneficial effects in $\mathrm{AD}$ related in vivo and in vitro models (Wang et al., 2015; Zhu et al., 2016). The conversion from dietary FAs to $\omega-3$ FAs, which are precursors of SPMs has been reported to be decreased in the liver of $\mathrm{AD}$ patients (Kang and Rivest, 2012). Accordingly, we have previously found that the levels of SPMs were lower in different areas of the postmortem $\mathrm{AD}$ brains including the hippocampus and the entorhinal cortex (Lukiw et al., 2005; Wang et al., 2015). Interestingly, results from clinical trials using PUFAs to treat $\mathrm{AD}$ patients showed that $\omega-3$ FAs treatment has beneficial effects only on the patients with mild cognitive impairment (MCI) (YurkoMauro et al., 2010) but not on late stage AD patients. Therefore, it is plausible that SPMs are the effective factors mediating the protective effects of $\omega-3 \mathrm{FAs}$, however, the conversion from
FAs to SPMs is decreased in late stage AD patients. Hence, we hypothesized that SPMs treatment is more effective for $\mathrm{AD}$ patients. Afterward, we tested this hypothesis on AD related cellular models including neuronal and microglia models and observed that all the types of SPMs could improve neuronal survival, and MaR1 was more effective in microglial phagocytosis of amyloid- $\beta(A \beta)_{42}$ (Zhu et al., 2016), indicating that inducing inflammation resolution by SPMs especially by MaR1 could be a novel therapeutic strategy for AD. MaR1 synthesis is initiated by the 14-lipoxygenation of DHA to yield 14S-hydro(peroxy)$4 \mathrm{Z}, 7 \mathrm{Z}, 10 \mathrm{Z}, 12 \mathrm{E}, 14 \mathrm{~S}, 16 \mathrm{Z}, 19 \mathrm{Z}$-docosahexaenoic acid and then to 13S, 14S-e MaR. This intermediate is then enzymatically hydrolyzed to MaR1 (Deng et al., 2014; Dalli et al., 2016). The biological functions of MaR1 have been showed in various disease models: MaR1 has to stimulate the pro-inflammatory M1 to anti-inflammatory M2 macrophage phenotype shifts and tissue regenerative actions of MaR1 have also been reported (Dalli et al., 2013). Moreover, MaR1 has been reported suppressed oxidative stress in a left pulmonary hilum I/R mouse model (Sun et al., 2017).

However, the effects of MaR1 on AD animal models have not been studied, and the mechanisms underlying the protective effects of MaR1 remain less understood. The aim of this study was to investigate the effects of MaR1 on behavioral deficits and pathological changes induced by intra-hippocampal injection of $A \beta_{42}$ protein in a mouse model along with the molecular mechanisms of action of MaR1.

\section{MATERIALS AND METHODS}

\section{Animals}

Forty C57BL/6 mice (male, 3-4 months old, weight $26-31 \mathrm{~g}$ ) were obtained from animal experiment center of Jilin University. The animals were housed at controlled room temperature $\left(22 \pm 2^{\circ} \mathrm{C}\right)$ and humidity $(50-60 \%)$ under a $12 \mathrm{~h}: 12 \mathrm{~h}$ light-dark cycle. Free access to water and food was provided. All procedures used in the present study followed the "National Institutes of Health Guide for Care and Use of Laboratory Animals" (Publication No. 85-23, revised 1985) and were approved by the Animal Ethics Committee of Jilin University. Efforts were also made to minimize animal suffering and to reduce the number of animals used.

\section{A 342 and MaR1 Preparation}

$\mathrm{A} \beta 42$ (Abcam, United Kingdom, ab120301) was prepared as a stock solution at a final concentration of $1 \mathrm{mg} / \mathrm{ml}$ in sterile 0.1 $\mathrm{M}$ phosphate-buffered saline (PBS), and aliquots were stored at $-20^{\circ} \mathrm{C}$. A $\beta 42$ solution was aggregated by incubation at $37^{\circ} \mathrm{C}$ for 4 days before use. MaR1 (Cayman Chemical, United States) was dissolved in alcohol at the concentration of $0.1 \mu \mathrm{g} / \mu \mathrm{l}$ and was further diluted with PBS to $0.01 \mu \mathrm{g} / \mu 1$ solution before use.

\section{Primary Culture of Microglia}

Mouse brain mixed glial cells were prepared from whole brains of 1-3 day postnatal C57BL6/J mice and dissociated with a mild mechanical trituration. Cells were seeded in the cell culture 
bottles $\left(75 \mathrm{~cm}^{2}\right)$ pre-coated with poly-D-lysine. The culture medium was DMEM-High glucose supplemented with $10 \%$ fetal bovine serum, $1 \%$ penicillin/streptomycin (all from Life Sciences, United States) and $0.8 \%$ insulin. Microglia were further extracted from the mixed glial cell cultures by mild trypsinization digestion method as previously described (Saura et al., 2003).

\section{HT22 Cell Culture}

HT22 mouse hippocampal neuronal cell line were cultured at $37^{\circ} \mathrm{C}$ in Dulbecco's modified Eagle's medium (DMEM) supplied with 10\% fetal bovine serum (FBS) and antibiotic solution (100 U/ml penicillin, $0.1 \mathrm{mg} / \mathrm{ml}$ streptomycin) in a humidified atmosphere in the presence of 5\% CO2 until they reached confluence, when reached $90 \%$ confluence, cells were subcultured after treatment with $0.25 \%$ trypsin-EDTA mixture.

\section{Cell Viability Assay}

Cell Counting Kit-8 (CCK-8, meilunbio) was used to assess the viability of HT22 neuronal cell and microglia. Cells were seeded in 96-well plates and cultured for $24 \mathrm{~h}$ (5000/well). After treatment with different concentrations of MaR1 $(0.005 \mu \mathrm{M}$ MaR1, $0.05 \mu \mathrm{M}$ MaR1, $0.5 \mu \mathrm{M}$ MaR1) for $6 \mathrm{~h}, 10 \mu \mathrm{L}$ CCK-8 solution were added into each well. The optical density values were measured by microplate reader (Bio-Rad iMark) at $490 \mathrm{~nm}$.

\section{Stereotaxic Intra-Hippocampal A 442 Injection and Drug Treatments}

Mice were randomly divided into 4 groups: (1) Vehicle group $(n=10)$ : bilateral injections of $1 \mu \mathrm{l}$ PBS in the hippocampus and intra-cerebroventricular injection of $1 \mu \mathrm{l}$ solvent of MaR1; (2) A $\mathrm{\beta} 42$ group $(n=10)$ : bilateral hippocampal injections of $1 \mu \mathrm{l} A \beta 42$ solution and intra-cerebroventricular injection of $1 \mu \mathrm{l}$ solvent of MaR1; (3) MaR1 group ( $n=10)$ : bilateral hippocampal injections of $1 \mu \mathrm{l}$ PBS and intra-cerebroventricular injection of $1 \mu \mathrm{l} \mathrm{MaR} 1$ solution; and (4) A $342+\mathrm{MaR} 1$ group $(n=10)$ : bilateral hippocampal injections of $1 \mu \mathrm{l} A \beta 42$ solution and intracerebroventricular injection of $1 \mu \mathrm{l} \mathrm{MaR} 1$ solution.

Mice were anesthetized with isoflurane and the heads were fixed on a stereo locator. A volume of $1 \mu \mathrm{l}$ of MaR1 solution or MaR1 solvent was injected into the ventricle of mouse brains at the following stereotaxic coordinates using a microsyringe: anteroposterior, $-0.3 \mathrm{~mm}$ from bregma; mediolateral, $+1.00 \mathrm{~mm}$ from midline; and dorsoventral, $-2.2 \mathrm{~mm}$ from dura. The needle was removed after 20 min using three intermediate steps with a 1 min inter-step delay to minimize backflow.

Then, the same procedure was used for bilateral hippocampal injections of $A \beta 42$. A volume of $1 \mu$ l of $A \beta 42$ solution or PBS was injected into each side at the following stereotaxic coordinates: anteroposterior, $-2.46 \mathrm{~mm}$ from bregma; mediolateral, $\pm 1.5 \mathrm{~mm}$ from midline; and dorsoventral, $-2.0 \mathrm{~mm}$ from dura. The needle was removed after $5 \mathrm{~min}$. Mice were placed on a thermal pad $\left(32-33^{\circ} \mathrm{C}\right)$ till they were awake.

\section{Morris Water Maze (MWM) Test}

To assess the memory and spatial learning ability of mice, MWM test was conducted 7 days after $A \beta 42$ and MaR1 administration. The experimental apparatus consisted of a circular tank (diameter $=100 \mathrm{~cm}$, height $=50 \mathrm{~cm}$ ) that was divided into four quadrants. It was filled with water and was maintained at $22 \pm 2^{\circ} \mathrm{C}$. A platform (diameter $=9 \mathrm{~cm}$ ) was placed in the pool approximately $1.0 \mathrm{~cm}$ below the surface of the water in one of the four quadrants. On the four sides of MWM apparatus, different shapes of visual cues were placed on the inside wall of the pool in a diagonal pattern. A non-toxic white food additive titanium dioxide was added to the water in the pool to contrast with the mice color. We used tracking software (Viewer 2 Tracking Software, Ji Liang Instruments, China) to monitor and record each trial. The MWM paradigm consisted of four $90 \mathrm{~s}$ trials per day for 5 consecutive days followed by the probe test on the sixth day. At the end of each trial, the mice were dried with a dry cloth and returned back to the home cages, which were kept on a thermal pad. The interval between each trial for a mouse was more than $30 \mathrm{~min}$. For each trial, starting position was different and the mice were allowed to swim while being tracked by the software. The trial ended when the animal reached the platform or when $90 \mathrm{~s}$ had elapsed. If the mouse did not reach the platform, it was directed and placed onto the platform for $10 \mathrm{~s}$. A $90 \mathrm{~s}$ probe trial was performed on the sixth day to determine memory retention. For this single trial, the submerged platform was removed and each mouse was placed into the quadrant opposite to the quadrant that formerly contained the platform. The number of platform crossing, the time spent in the quadrant containing the platform previously, and escape latency were recorded.

\section{Immunohistochemistry}

Mice were deeply anesthetized with isoflurane and were sacrificed 1 day after they took WMW test. The brains were harvested, fixed in paraformaldehyde, and then saturated with increasing sucrose concentrations $(10 \%, 20 \%, 30 \%)$ in PBS. Brains were frozen and sectioned coronally at $10 \mu \mathrm{m}$ thickness using a microtome. Fixed sections were incubated at room temperature for $1 \mathrm{~h}$ in 5\% normal goat serum in PBS, followed by overnight incubation at $4^{\circ} \mathrm{C}$ with Iba- 1 antibody (1:100; Abcam, ab178847) for microglia and GFAP antibody (1:300; Cell Signaling, 3670) for astrocytes. Thereafter, the sections were washed three times with PBS and incubated for $1 \mathrm{~h}$ with Goat Anti-Rabbit IgG (1:500; Abcam, ab150080) and Goat Anti-Mouse IgG (1;500, Abcam, ab150113). Sections were then rinsed with PBS three times. For nucleus labeling, the sections were incubated with DAPI (1:1000; Beyotime, C1006). Positive cells were quantified under a Laser scanning confocal microscope in stained sections in the hippocampus and cortex.

ImageJ software (NIH, Bethesda, MD, United States) was used to measure Iba- 1 and GFAP immune-positive staining in the CA1 region and cortex. Four sections per brain were analyzed, and multiple images were taken within each section to cover the CA1 region and cortex. Threshold was equally adjusted across all brain sections to highlight the stained area, and particles were analyzed to determine the total stained area. Results were expressed as total area stained per total area analyzed (area fraction). 


\section{Fluoro-Jade B (FJB) Staining}

Prior to immunostaining, fixed sections described above were mounted onto slides from distilled water and then air-dried for at least $30 \mathrm{~min}$ on a slide warmer at $50^{\circ} \mathrm{C}$. Then, the tissue sections were immersed in a basic alcohol solution of $1 \%$ sodium hydroxide in $80 \%$ ethanol for $5 \mathrm{~min}$. They were then rinsed in $70 \%$ ethanol for $2 \mathrm{~min}$, followed by distilled water for $2 \mathrm{~min}$, and then incubated in $0.06 \%$ potassium permanganate solution for $10 \mathrm{~min}$. After a water rinse for 1-2 min, the slides were then transferred to a $0.0004 \%$ solution of Fluoro-Jade B (EMD Millipore, United States) dissolved in $0.1 \%$ acetic acid for $20 \mathrm{~min}$. The slides were then rinsed thrice in distilled water, ensuring that each rinse lasted for $1 \mathrm{~min}$. The slides were dried by paper towel and then air-dried on a slide at $50^{\circ} \mathrm{C}$ for at least $5 \mathrm{~min}$. The airdried slides were then immersed in xylene for at least $1 \mathrm{~min}$ and coverslipped using DPX mounting medium. FJB-positive cells were quantified under a microscope (Olympus BX51, Japan) in stained sections in the hippocampus and cortex. Four sections per animal were viewed. Results were expressed as the number of FJB positive cells per section.

\section{Tissue Processing for Western Blot and Cytometric Beads Array (CBA)}

For western blot and CBA, anesthetized mice were subjected to cervical dislocation, the hippocampus and cortex were dissected out and immediately frozen in liquid nitrogen. Proteins were extracted in 10X volume/weight with radio-immuno precipitation assay (RIPA) buffer, supplemented with $1 \%$ protease inhibitor cocktail and $1 \%$ phosphatase inhibitor cocktail (SigmaAldrich, United States), and centrifuged at $12,000 \mathrm{rpm}$ at $4^{\circ} \mathrm{C}$ for $20 \mathrm{~min}$. The supernatant was collected and stored for further analyses.

\section{Cytometric Beads Array (CBA)}

The levels of tumor necrosis factor (TNF)- $\alpha$, interleukin (IL)-2, IL-4, IL-6, IL-10, Interferon (IFN)- $\gamma$ and IL-17A in the hippocampus and cortex tissues were analyzed by $\mathrm{CBA}$ (BD Biosciences, United States) according to the manufacturer's manual. Cytokine levels were then quantified using flow cytometry.

\section{Enzyme-Linked Immunosorbent Assay (ELISA)}

The cortex and hippocampus of mice were homogenized with 10X volume/weight normal saline. The levels of monocyte chemoattractant protein (MCP)-1 were measured by ELISA kit (Shanghai Yuan-ye Bioengineering Institute, China) according to the manufacturer's manual.

\section{Western Blot}

Analysis of proteins by western blot was performed using proteins extracted from the hippocampus. Total protein concentration was determined by a BCA assay kit. Briefly, samples containing $40 \mu \mathrm{g}$ protein each were mixed with an equal volume of $2 \mathrm{X}$ Laemmli sample buffer, and were boiled at $95^{\circ} \mathrm{C}$ for $5 \mathrm{~min}$. The denatured samples were then loaded on a
$10 \%$ SDS-PAGE gel, after which the proteins were transferred to a nitrocellulose membrane under $85 \mathrm{~mA}$ current overnight at $4{ }^{\circ} \mathrm{C}$. The membranes were blocked with $5 \%$ non-fatty dry milk at room temperature for $45 \mathrm{~min}$, and then incubated with the following antibodies: anti-actin (1:2500; Abcam, ab8224), anti-LaminA (1:1000; Abcam, ab26300), Anti-GAPDH (1:10000, Abcam, ab181602), anti-phospho-PI3K (1:1000; Abcam, ab182651), anti-PI3K (1:1000; Abcam, ab191606), anti-phospho-AKT (1:5000; Abcam, ab81283), anti-AKT (1:2000; Abcam, ab28422), anti-phospho-p38 (1:1000; Abcam, ab195049), anti-p38 (1:2000; Abcam, ab170099), anti-phosphoERK1 + ERK2 (1:1000; Abcam, ab201015), anti-ERK1 + ERK2 (1:10000; Abcam, ab184699), anti-caspase 3 (1:5000; Abcam, ab184737), anti-phospho-mTOR (1:1000; Cell signaling, 2971), anti-mTOR (1:1000; Cell signaling, 2972), anti-LC3B (1:3000; Abcam, ab51520), Anti-SQSTM1/p62 (1:500; Abcam, (ab56416) Anti-Beclin 1 (1:2000, Abcam, ab62557), in Tris-buffered saline with $0.1 \%$ Tween 20 (TBS-T) at $4^{\circ} \mathrm{C}$ overnight. After incubation with appropriate secondary antibody, the antigen-antibody complexes were visualized with the ECL chemiluminescence system (Amersham, United Kingdom). The relative densities of bands were analyzed using the ImageJ software.

\section{Statistical Analysis}

All values are expressed as the mean \pm SEM. Normal distributions and homogeneity of variance were found for all analyzed categories. All statistical analyses were conducted using GraphPad Prism 7 software (GraphPad Software, La Jolla, CA, United States). Independent $t$-test was used to compare between the two groups, and one-way-ANOVA was used in multi-group pairwise comparison. In all instances, statistical significance was defined as follows: ${ }^{*} P<0.05,{ }^{* *} P<0.01,{ }^{* * *} P<0.001$.

\section{RESULTS}

\section{MaR1 Ameliorated A $\beta 42$ Protein-Induced Cognitive Decline}

The escape latency for all the groups gradually decreased during 5 consecutive training days, as indicated by Figures $\mathbf{1 A}, \mathbf{B}$. Differences gradually became larger in the mean latency between the 4 groups. On the fifth day, the escape latency of $A \beta 42$ group was significantly longer than that of Vehicle group $(P<0.05)$, and the escape latency of $A \beta 42+$ MaR1 group was significantly shorter than that of $\mathrm{A} \beta 42$ group $(P<0.05)$. There was no significant difference in escape latency between Vehicle group and MaR1 group. In the probe test, the number of platform crossing and the time spent in the target quadrant by the $\mathrm{A} \beta 42$ group were significantly less than those in Vehicle group $(P<0.001)$, and the number of platform crossing and the time spent in the target quadrant by the $\mathrm{A} \beta 42+\mathrm{MaR} 1$ group were more than those in $\mathrm{A} \beta 42$ group $(P<0.05)$. There was no significant difference between Vehicle group and MaR1 group in the platform crossing and the time spent in the target quadrant (Figures 1C,D). 
A
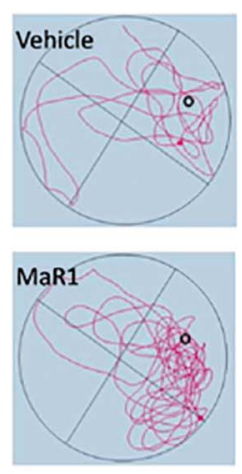

C

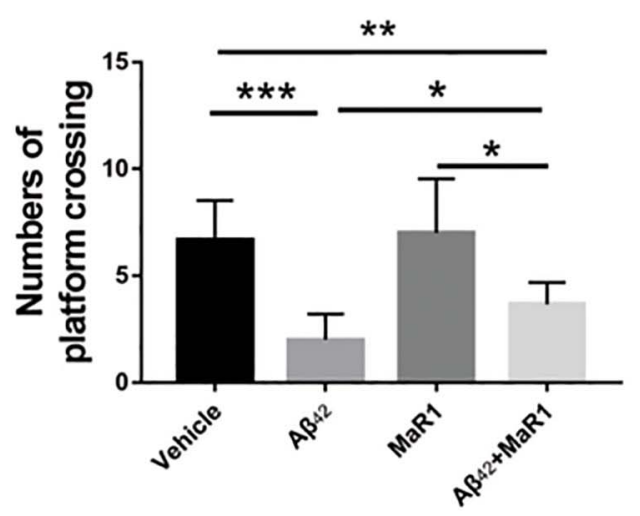

B

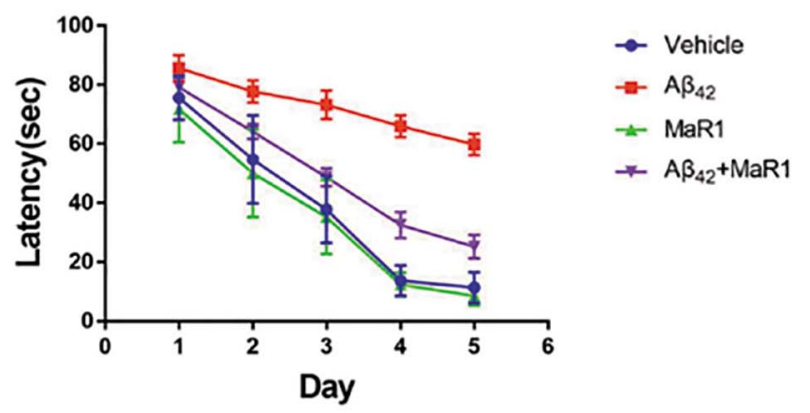

D

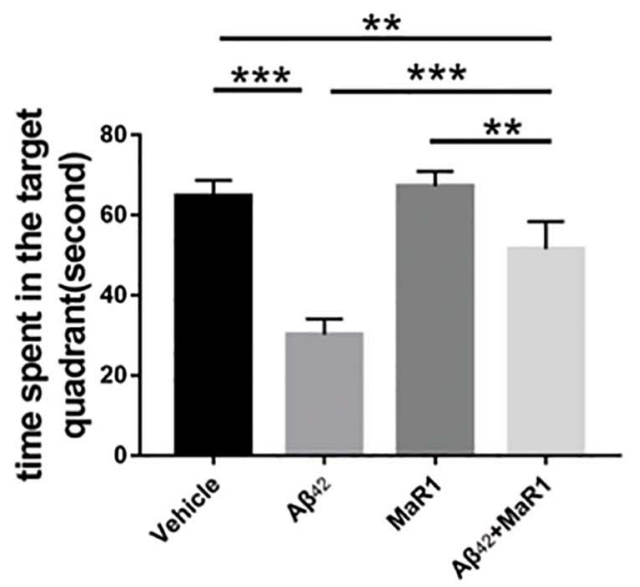

FIGURE 1 | MaR1 improved learning and memory deficits in $A \beta_{42}$ treated mice. (A) Typical swimming tracks of the four groups on the sixth day after drug treatment. (B) Escape latency gradually decreased with time in all the four groups. On the sixth day after drug treatment, $A \beta_{42}$ treatment group showed significantly increased escape latency compared to Vehicle group, however, $A \beta_{42}+$ MaR1 treatment reversed the changes induced by $A \beta_{42}(P<0.05)$. (C) Numbers of platform crossing on the sixth day after drug treatment in probe test. $A \beta_{42}$ treatment significantly decreased the number of platform crossing compared to Vehicle group, while $A \beta_{42}+$ MaR1 treatment attenuated the changes induced by $A \beta_{42}(P<0.05)$. (D) Time spent in the target quadrant in the probe test on the sixth day after drug treatment. $A \beta_{42}$ treatment significantly decreased the time spent in the target quadrant compared to Vehicle group, while $A \beta_{42}+$ MaR1 treatment attenuated the changes induced by $A \beta_{42}(P<0.001)$. Data are expressed as mean \pm SEM, 10 mice in each group, and statistical significance is defined as follows: $* P<0.05$, ${ }^{* *} P<0.01,{ }^{* * *} P<0.001$

\section{MaR1 Attenuated Neuronal Degeneration and Glia Activation in the Hippocampus and Cortex of Mice Treated With A $\beta 42$}

Neurodegenerative neurons were revealed by FJB staining (Figure 2). In the hippocampus and cortex, the number of degenerative neurons in $A \beta 42$ group was more than that in Vehicle group $(P<0.001)$, while the number of degenerative neurons in $\mathrm{A} \beta 42+\mathrm{MaR} 1$ group was less than that in $\mathrm{A} \beta 42$ group $(P<0.001)$. No significant difference was observed in the number of degenerative neurons between Vehicle group and MaR1 group.

Iba-1 and GFAP were used to demonstrate microglia and astrocytes, respectively (Figure 3). In the hippocampus and cortex, the percentage of area with Iba-1 and GFAP positive staining cells in $A \beta 42$ group was higher than that in Vehicle group $(P<0.001)$, and the percentage of area with Iba-1 and GFAP positive staining cells in $\mathrm{A} \beta 42+\mathrm{MaR} 1$ group was lower than that in $\mathrm{A} \beta 42$ group $(P<0.001)$. There was no significant difference in the percentage of area with Iba-1 or GFAP positive staining cells between Vehicle group and MaR1 group.

\section{MaR1 Reduced Pro-inflammatory Cytokine and Chemokine Production and Increased Anti-inflammatory Cytokine Production}

The production of different cytokines was examined to further investigate the effect of MaR1 on neuroinflammation in $\mathrm{AD}$. The levels of TNF- $\alpha$, IL- 6 and MCP- 1 in A $\beta 42$ group were significantly higher than those in Vehicle group $(P<0.05)$, while treatment of $\mathrm{A} \beta 42+\mathrm{MaR} 1$ significantly decreased the levels of TNF- $\alpha$, IL- 6 and MCP-1 induced by A $\beta 42(P<0.05)$. There was no significant difference in TNF- $\alpha$, IL- 6 and MCP- 1 between Vehicle group and MaR1 group (Figures 4A-F). The levels of IL- 2 and IL-10 in A $\beta 42$ group were significantly higher than those in Vehicle group in the hippocampus and cortex $(P<0.05)$; 


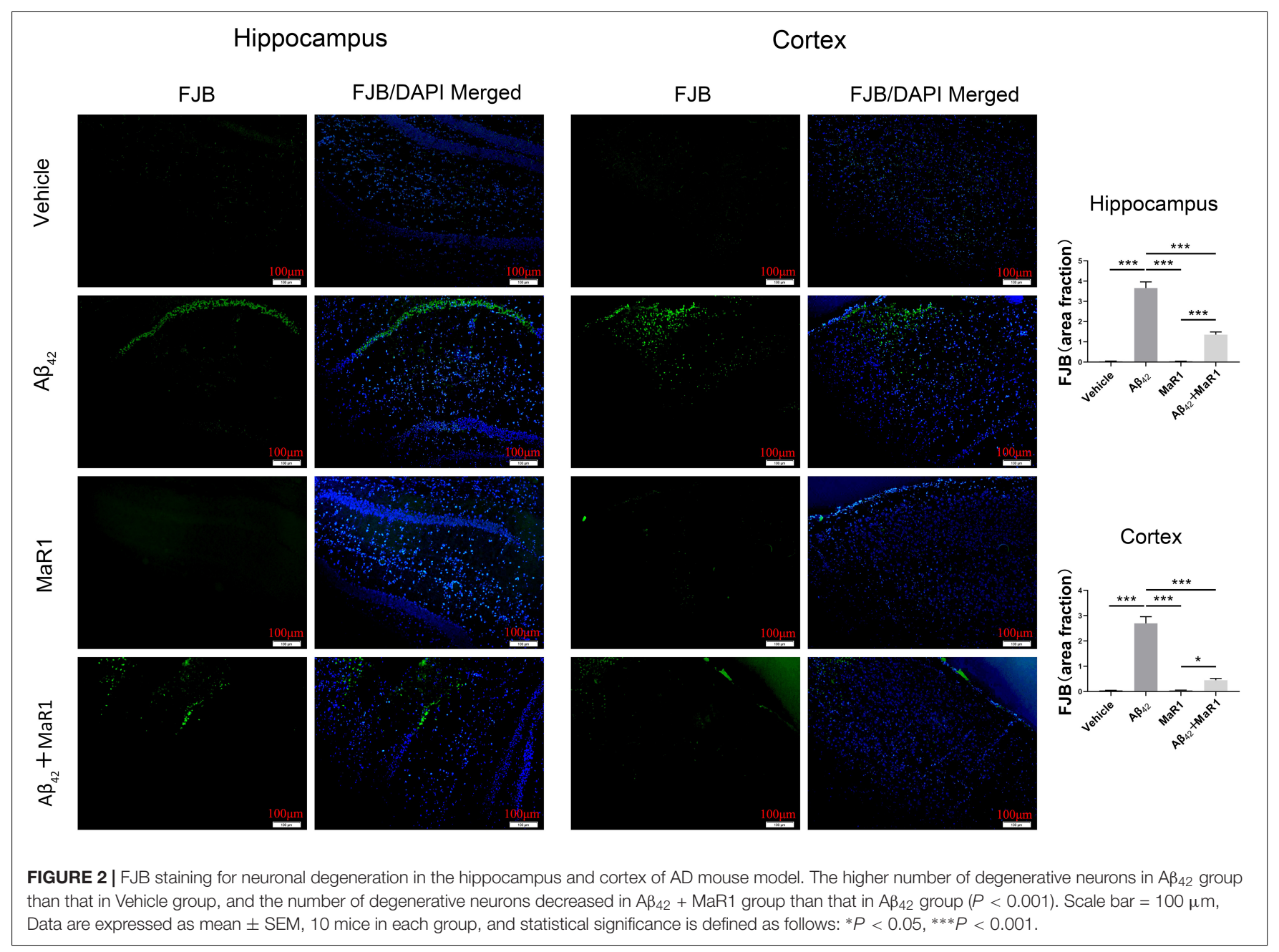

MaR1 treatment increased the production of IL-2 and IL-10 in the hippocampus $(P<0.05)$; the levels of IL-2 and IL-10 in $\mathrm{A} \beta 42+\mathrm{MaR} 1$ group were higher than those in Vehicle group in the cortex but not in the hippocampus $(P<0.05)$ (Figures $4 \mathrm{G}-\mathbf{J}$ ). No difference was observed in the levels of IL-4, IFN- $\gamma$ and IL-17A among the four groups (data not shown).

\section{MaR1 Exerted Neuroprotection and Suppressed Neuroinflammation by Modulating Autophagy, Apoptosis, and MAPK Signal Pathways}

Compared with Vehicle group, the ratio of $\mathrm{p}-\mathrm{PI} 3 \mathrm{~K} / \mathrm{t}-\mathrm{PI} 3 \mathrm{~K}$ and p-AKT/t-AKT were down-regulated (Figures 5A,B), while the ratio of $\mathrm{p}$-mTOR/t-mTOR, p-p38/t-p38, as well as the levels of caspase 3 were up-regulated in $\mathrm{A} \beta 42$ group $(P<0.01)$ (Figures 5C-E), however, these $\mathrm{A} \beta 42$ induced changes were reversed by $\mathrm{A} \beta 42+$ MaR1 treatment $(P<0.05)$. The ratio of p-ERK/t-ERK was increased by both, A $\beta 42(P<0.001)$ and MaR1 $(P<0.01)$ alone, and was further increased by their costimulation as compared to Vehicle group $(P<0.01)$ and $\mathrm{A} \beta 42$ group $(P<0.05)$ (Figure 5F).

\section{MaR1 Exerted Neuroprotection and Suppressed Neuroinflammation by Modulating Autophagy Signal Pathways}

Compared with Vehicle and MaR1 group, the ratio of LC3II/LC3-I was down-regulated $(P<0.01, P<0.05)$, while the levels of p62 was up-regulated in $\mathrm{A} \beta_{42}$ group $(P<0.01)$, however, these $\mathrm{A} \beta_{42}$ induced changes were reversed by $\mathrm{A} \beta_{42}+$ MaR1 treatment $(P<0.01)$. Compared with MaR1 group, the level of Beclin1 was decreased in $\mathrm{A} \beta_{42}$ group $(P<0.01)$, and increased by $\mathrm{A} \beta_{42}+$ MaR1 treatment $(P<0.01)$ (Figure 6).

\section{In vitro Cytotoxicity Analysis}

The cell proliferation rate was at a normal level in HT22 neuronal cell and microglia after MaR1 treatment. There was no significant difference in cell proliferation after treatment with different MaR1 concentrations (Figure 7).

\section{DISCUSSION}

We have previously showed that inflammation resolution is impaired in the $\mathrm{AD}$ brains and stimulating resolution 


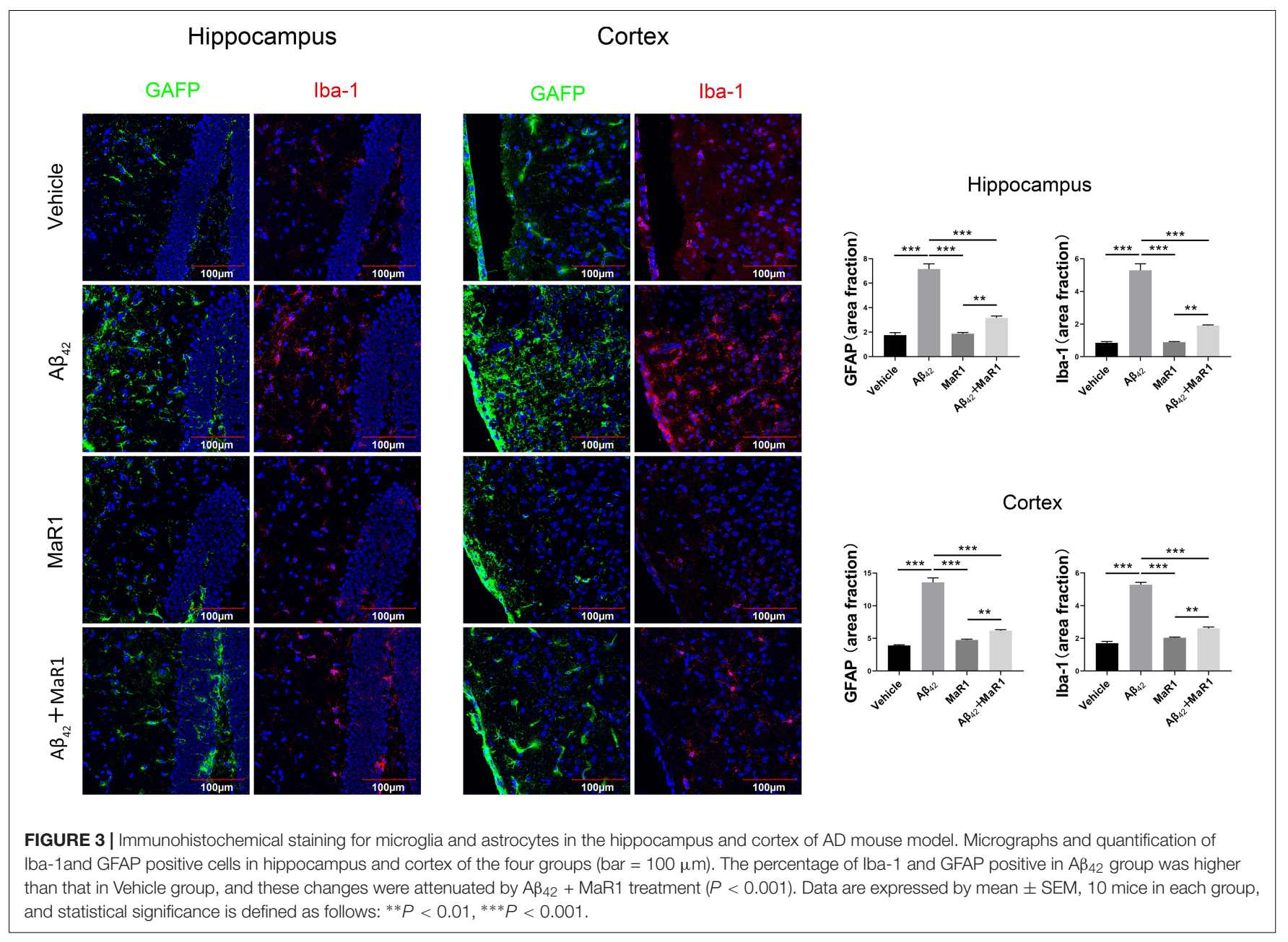

of inflammation by SPMs has proved to be beneficial in cellular models of $\mathrm{AD}$, therefore, indicating that resolution of inflammation by SPMs may be a potential therapeutic target for AD (Wang et al., 2015; Zhu et al., 2016). Aspirin-triggered LXA4 (ATL) has been shown to alleviate AD related pathological changes and improve the cognitive impairment in $\mathrm{AD}$ animal models (Medeiros et al., 2013; Dunn et al., 2015). However, there is no study regarding the effects of SPMs on in vivo AD models. In this study, we have investigated the effects of MaR1 on the behavioral and pathological changes as well as the molecular pathways affected by MaR1 in AD mouse model.

Administrating $A \beta_{42}$ peptide to hippocampus can induce behavioral changes specific to learning and memory (Colaianna et al., 2010; Passos et al., 2010). In the MWM tests, a decrease in the escape latency in all the four groups was observed as the training proceeded, indicating an ongoing spatial learning and memory in the mice. However, the escape latency in $A \beta_{42}$ group was significantly longer compared with Vehicle group on the fifth day, suggesting that mice treated with $A \beta_{42}$ developed learning and memory deficits. But co-stimulation with MaR1 and $A \beta_{42}$ ameliorated the cognitive decline indicated by our observation that the escape latency in $A \beta_{42}+$ MaR1 group was shorter than that in $A \beta_{42}$ group on the fifth day. Similar trends were also found in the probe test, as the number of platform crossings and the time spent in the target quadrant by $\mathrm{A} \beta_{42}+\mathrm{MaR} 1$ group were longer than those in $A \beta_{42}$ group. All these results suggest that MaR1 could improve behavioral dysfunction induced by $A \beta_{42}$. We also noticed that there were no significant differences in escape latency, cross times, and time spent in target quadrant between Vehicle and MaR1 group. The possible explanation might be that MaR1 alone does not have any effect on normal mice, however, it promotes inflammation resolution only when there are ongoing inflammatory changes.

Consistent with the results of behavioral test, the injection of $\mathrm{A} \beta_{42}$ into hippocampus caused neuronal degeneration revealed by FJB staining, which resulted in memory deficits. The number of degenerative neurons in the hippocampus and cortex of $\mathrm{A} \beta_{42}$ and MaR1 co-stimulation group was significantly lower than that of $A \beta_{42}$ group, suggesting that MaR1 could restore the behavioral deficits by protecting the neurons from $A \beta_{42}$ toxicity. Increased number of microglia and astrocytes have been observed in postmortem AD brain (Medeiros et al., 2013). Moreover, it has been demonstrated that activation of glia cells including microglia and astrocytes by $\mathrm{A} \beta$ leads to elevated production of pro-inflammatory mediators, chronic inflammation, neurodegeneration, decreased glutamate uptake, 
A Hippocampus

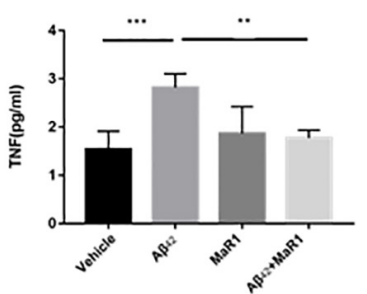

E

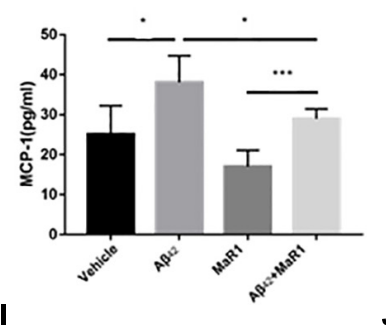

I

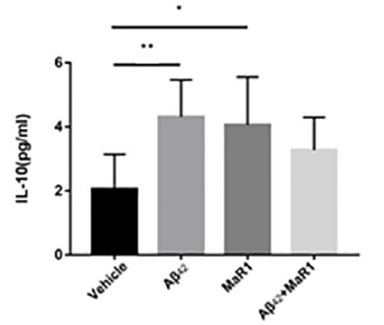

B

Cortex

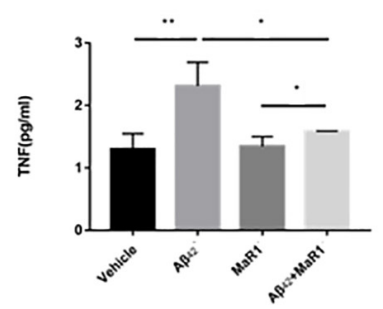

$\mathbf{F}$

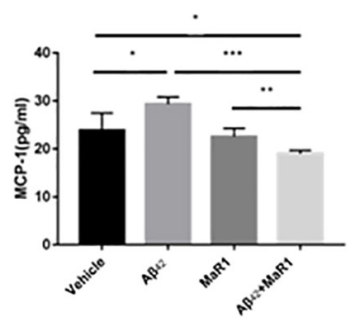

J

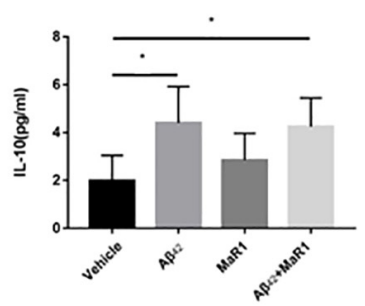

C

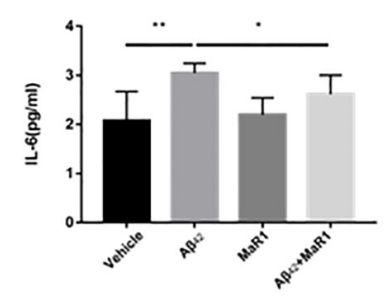

G

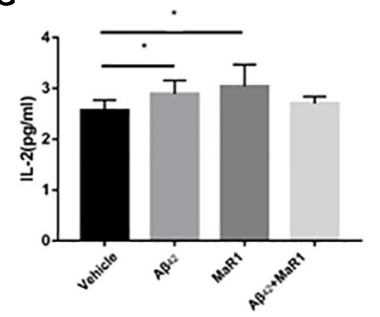

D

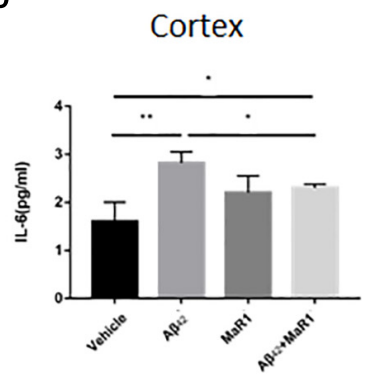

H

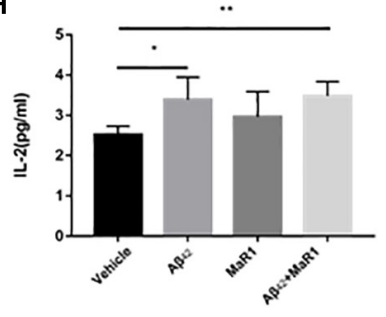

FIGURE 4 | Effects of MaR1 on the secretion of inflammatory cytokines. ELISA and CBA analysis for different inflammatory mediators in the hippocampus and cortex, TNF (A,B), IL-6 (C,D), MCP-1 (E,F), IL-2 (G,H), IL-10 (I,J). (A-F) The levels of TNF- $\alpha$, IL-6 and MCP-1 in A $\beta_{42}$ group were significantly higher than those in Vehicle group $(P<0.05)$, while treatment of $\mathrm{A} \beta_{42}+$ MaR1 significantly decreased the levels of TNF- $\alpha$, IL-6 and MCP-1 induced by $A \beta_{42}(P<0.05)$. (G-J) The levels of IL-2 and IL-10 in $A \beta_{42}$ group were significantly higher than those in Vehicle group $(P<0.05)$; MaR1 treatment increased the production of IL-2 and IL-10 in the hippocampus $(P<0.05)$; the levels of IL-2 and IL-10 in A $\beta_{42}+$ MaR1 group were higher than those in Vehicle group in the cortex $(P<0.05)$. Data are expressed as mean \pm SEM, 10 mice in each group, and statistical significance is defined as follows: ${ }^{*} P<0.05,{ }^{* *} P<0.01,{ }^{* * *} P<0.001$.

loss of neuronal synapses, and ultimately cognitive deficits in AD (Prokop et al., 2013; Michaud and Rivest, 2015). In addition, increased cytokines can down-regulate the expression of $\mathrm{A} \beta$ phagocytosis receptors on microglia and ultimately result in insufficient microglial phagocytic activity (Hickman et al., 2008). The weakening of $A \beta$ clearance further aggravates the inflammatory response and forms a vicious circle. The immunohistochemical experiments showed that the number of Iba- 1 and GFAP positive cells were elevated after $A \beta_{42}$ treatment, however, this trend was reversed by combination treatment of $\mathrm{A} \beta_{42}$ and MaR1, suggesting that MaR1 could attenuate inflammation by inhibiting pro-inflammatory activation of microglia and astrocytes. We also observed that the level of MCP-1 was higher in the hippocampus and cortex of $A \beta_{42}$ injected mice, which decreased when mice received combined treatment of $A \beta_{42}$ and MaR1. Therefore, MaR1 may inhibit the chemotaxis of glia by decreasing the secretion of chemokines, which in turn reduces the inflammatory response. Fibrillar $A \beta$ in plaques has been shown to stimulate microglia to secret pro-inflammatory cytokines, including IL-1, IL-6 and TNF- $\alpha$ (Murphy et al., 1998; Combs et al., 2001). There are reports indicating that MaR1 reduces TNF- $\alpha$ and IL6 secretion in peripheral inflammation disease models such as septic mouse model (Hao et al., 2019), and human periodontal ligament cell model. In line with previous reports, we found MaR1 could reverse the increased levels of TNF$\alpha$ and IL- 6 induced by $A \beta_{42}$ in the central nervous system. The increased levels of IL- 6 , TNF- $\alpha$ are associated with increased $A \beta$ production, decreased $A \beta$ clearance, tau hyper phosphorylation (Ringheim et al., 1998; Zilka et al., 2012), synaptic dysfunction, and cognitive deficits (Singh-Manoux et al., 2014; Chang et al., 2017). In an aspergillus fumigatus keratitis mouse model, MaR1 was found to increase the expression of anti-inflammatory cytokine IL-10 (Tang et al., 2019). We also observed that both $A \beta_{42}$ and MaR1 could induce the secretion of anti-inflammatory cytokines, IL-2 and IL-10. It has been reported that IL-2 and IL-10 and their signaling pathways are elevated in the brain of $\mathrm{AD}$ patients (Strle et al., 2001; Gezen-Ak et al., 2013; Guillot-Sestier et al., 2015a). Some researchers hypothesize that the increase in IL-10 levels may lead to innate immunity repression and microglia dysfunction in $A \beta$ clearance (Guillot-Sestier et al., 


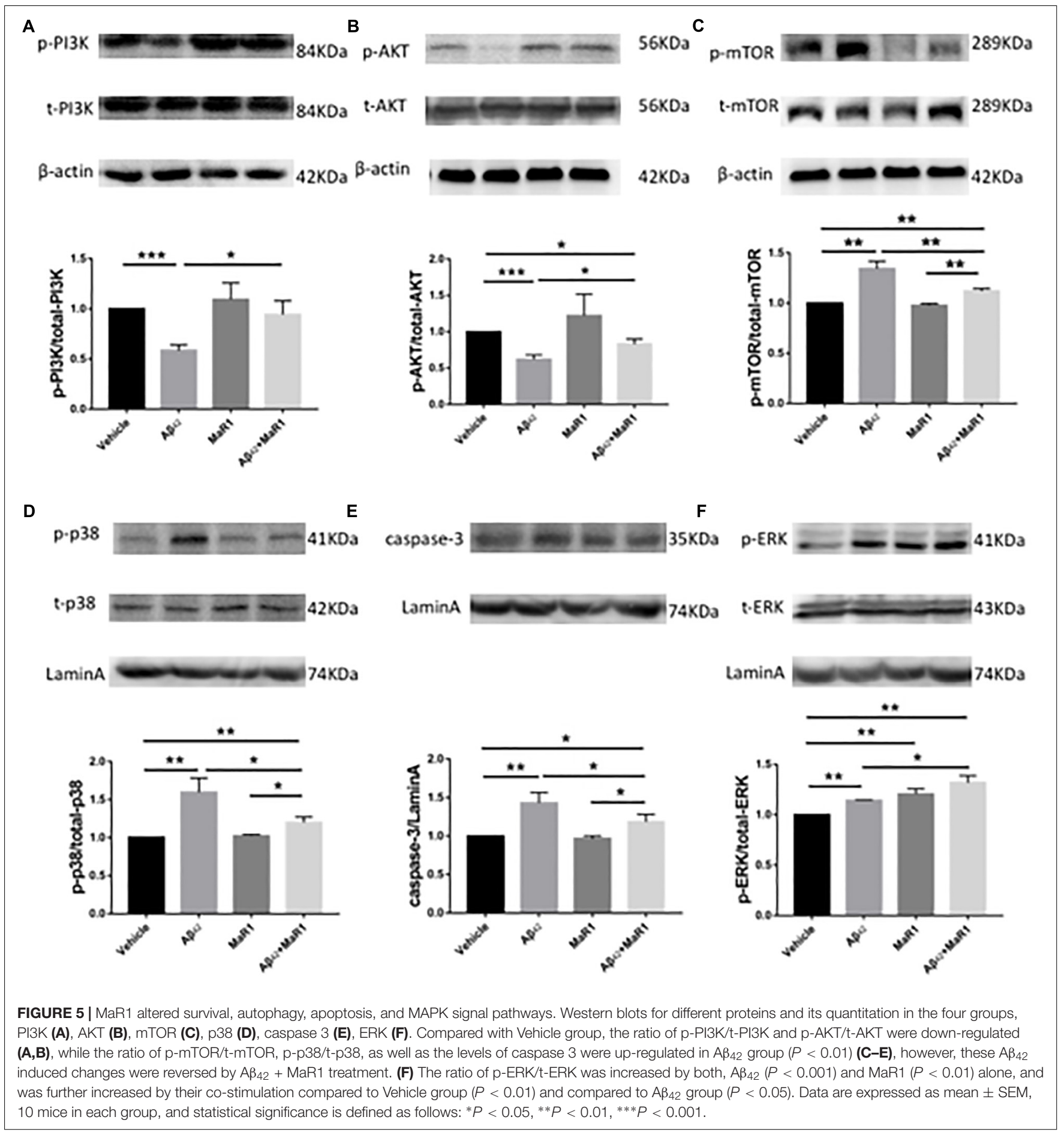

2015b), while the others argue that elevated anti-inflammatory cytokines levels is beneficial in decreasing amyloid plaque load, improving synaptic plasticity, and rescuing spine density (Dansokho et al., 2016; Alves et al., 2017). However, it may also be explained by third possibility that $A \beta_{42}$ induces inflammation in the brain, which in turn stimulates IL-10 secretion that plays an immunomodulatory role and facilitates the resolution of inflammatory cascades (Garcia et al., 2017). In summary,
MaR1 plays neuroprotective role by down-regulating proinflammatory activation of microglia and astrocytes, decreasing pro-inflammatory while increasing anti-inflammatory cytokines production, and promoting resolution of inflammation.

Even though beneficial effects have been reported in a different study using other SPMs in AD mice models (Zhu et al., 2016), there is no research that demonstrates the effects of MaR1 on $\mathrm{AD}$ animal models. Moreover, the underlying 

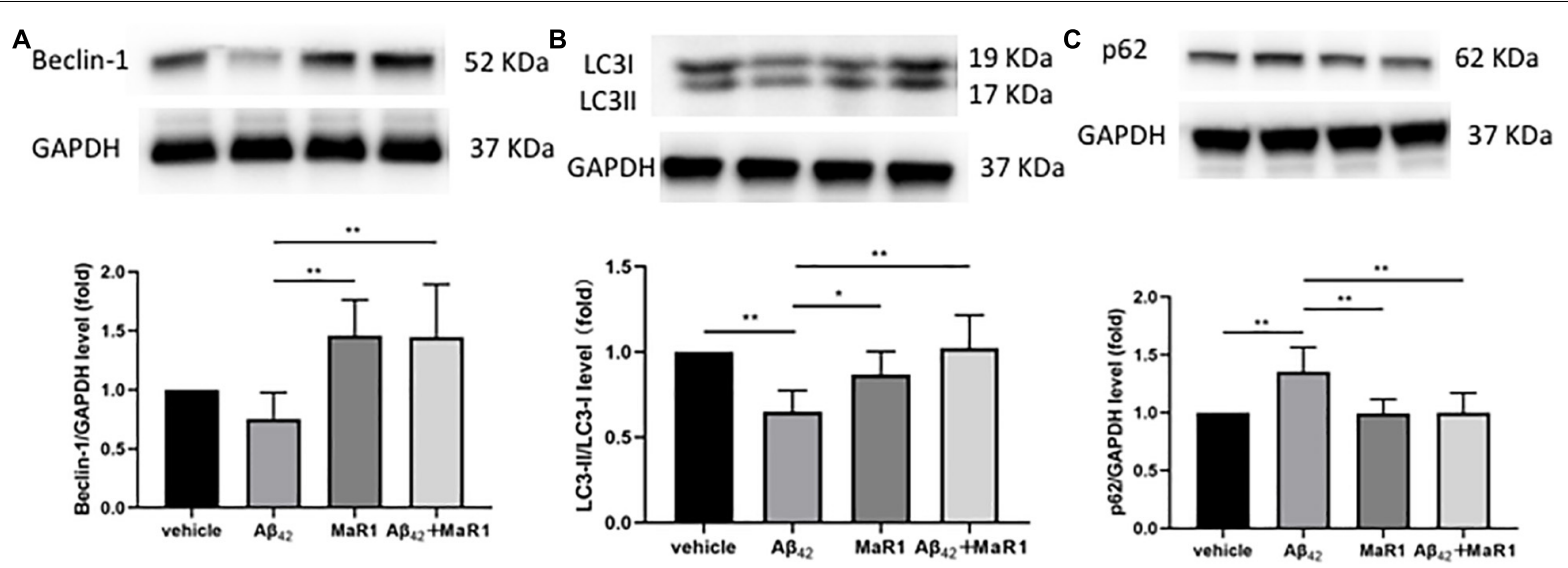

FIGURE 6 | MaR1 altered autophagy signal pathways. Compared with MaR1 group, the level of Beclin-1 (A) was decreased in A $\beta_{42}$ group $(P<0.01)$, and increased by $A \beta_{42}+$ MaR1 treatment $(P<0.01)$. Compared with Vehicle and MaR1 group, the ratio of LC3-II/LC3-I (B) was down-regulated $(P<0.01, P<0.05=$, while the levels of p62 (C) was up-regulated in $A \beta_{42}$ group $(P<0.01)$, however, these $A \beta_{42}$ induced changes were reversed by $A \beta_{42}+$ MaR1 treatment $(P<0.01)$. Data are expressed as mean \pm SEM, 10 mice in each group, and statistical significance is defined as follows: ${ }^{*} P<0.05,{ }^{* *} P<0.01$
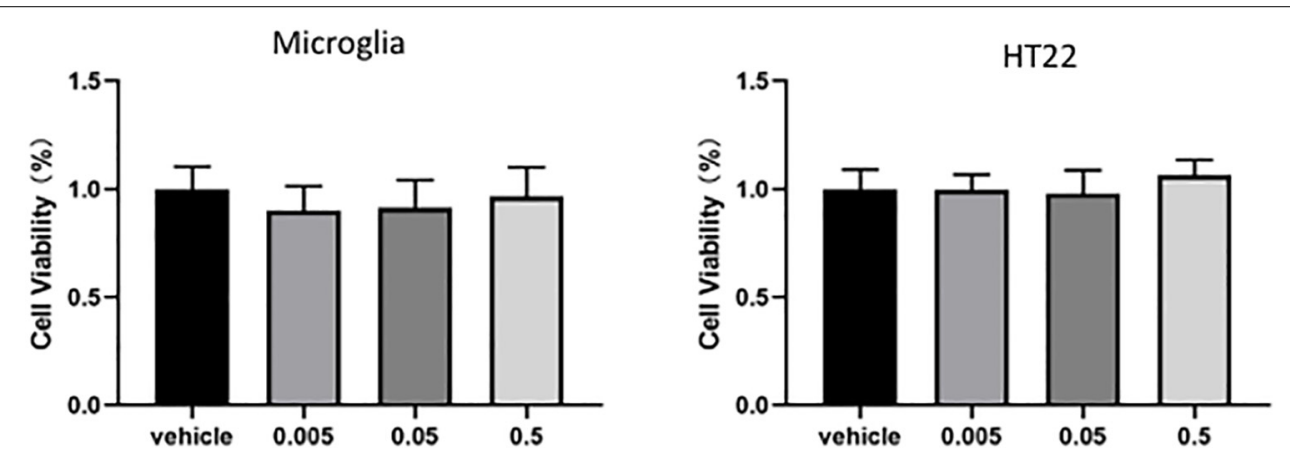

FIGURE 7 | In vitro cytotoxicity analysis. There was no significant difference in cell proliferation after treatment with different concentrations MaR1 both in HT22 and in microglia cells.

mechanisms are less understood and the receptor of MaR1 has not been identified. However, in this study, we observed that several signaling pathways involved in inflammation, cellular survival, proliferation, autophagy, apoptosis, and axon formation were altered by MaR1, including PI3K/AKT, ERK1/2, mTOR, $\mathrm{p} 38$, and caspase 3 .

PI3K/AKT signaling pathway plays an important role in cellular proliferation, growth, survival, motility, and metabolic functions (Kriplani et al., 2015). It has been demonstrated that PI3K/AKT can activate its downstream factors mTOR and Nrf2 (Kim et al., 2013), which are involved in axon branching, autophagy, alleviating oxidative damage, and down-regulating tau protein phosphorylation as well as apoptosis of neurons via inhibiting caspase 3 and glycogen synthase kinase (GSK) $-3 \beta$ (Chami et al., 2016). PI3K/AKT signaling has been found to be impaired in $\mathrm{AD}$ patients in previous studies (Wang et al., 2016), Besides AD, the same results have also been observed in other neurodegenerative diseases such as amyotrophic lateral sclerosis (ALS) and Parkinson's disease (PD) (Recabarren and Alarcon, 2017). All four types of SPMs have been reported to affect the expression of PI3K/AKT pathway and hence, play a protective role: LXA4 protected human or murine macrophages from apoptosis through activation of PI3K/AKT and ERK/Nrf2 pathways (Prieto et al., 2010); RvD1 reduced myocardial infarct size by increasing PI3K/AKT expression (Gilbert et al., 2015); NPD1 induced retinal pigment epithelial cell survival by activating PI3K/AKT signal pathway (Halapin and Bazan, 2010); MaR1 activated PI3K/AKT pathway to down-regulate Nedd4-2 protein and improve $\mathrm{Na}$, K-adenosine triphosphatase (ATPase) activity, and then stimulate alveolar fluid clearance (Zhang et al., 2017). In line with previous findings, we found that PI3K/AKT signaling was down-regulated in $A \beta_{42}$ induced $A D$ mouse model, while MaR1 administration enhanced PI3K/AKT signaling pathway and promoted cell survival, indicating that the protective role of MaR1 is partly due to activation of PI3K/AKT signaling pathway. Furthermore, similar to previous studies in humans and mouse models, we also observed that the mTOR signaling was enhanced in the $A \beta_{42}$ induced AD mouse model. The up-regulation of mTOR could result in reduction of autophagy, which probably is the main cause for abnormal protein aggregation in AD (O’Neill, 2013; Tramutola et al., 2017). This is further supported by studies that show that treatment 
with mTOR inhibitors improves the cognitive impairment in AD (Tramutola et al., 2017, 2018). Interestingly, previous studies have verified that precursors of SPMs, omega-3 PUFAs, are beneficial for chronic pathological conditions by inhibiting mTOR expression (Kim et al., 2018). In the present study, we observed that MaR1, the downstream product of PUFAs, could also down-regulate the elevated mTOR levels induced by $A \beta_{42}$ treatment, indicating that MaR1 could enhance autophagy by inhibiting mTOR pathway.

The reason for up-regulation of $\mathrm{mTOR}$ in $\mathrm{AD}$ maybe partly attributed to Ras/ERK, which is an up-stream activating factor for mTOR. Extracellular signal-regulated kinase (ERK) has been found to be elevated in the brain of $\mathrm{AD}$ patients (Russo et al., 2002). We observed that ERK signaling pathway was enhanced by both, $A \beta_{42}$ and MaR1 treatment alone or by their combination. Another mitogen-activated protein kinases (MAPKs) family member, p38 signaling pathway, was also enhanced by $A \beta_{42}$ treatment that was reversed by MaR1 treatment. ERK can activate AKT, hence it is the key regulator for neuronal plasticity and survival. In addition, ERK can also activate its downstream effector, cAMP response element binding protein (CREB) (Dineley et al., 2001; Bell et al., 2004), which is involved in synaptic plasticity. However, $\mathrm{A} \beta$-induced activation of ERK pathway in the hippocampus also leads to caspase activation (Chong et al., 2006) and aberrant hyper-phosphorylation of tau protein (Arora et al., 2015). Therefore, subsequent cellular response induced by ERK activation is complicated and can trigger both beneficial and detrimental effects on neuronal cells. The p38 MAPK activation leads to microglia and astrocyte activation and subsequently promotes neuroinflammation in AD (Hensley et al., 1999; Sun et al., 2003; Schnoder et al., 2016). Inhibition of p38 MAPK by p38 MAPK inhibitors can effectively alleviate chronic inflammatory diseases such as rheumatoid arthritis, cardiovascular disease, and inflammatory pain (Cohen et al., 2009; Marber et al., 2011; Arthur and Ley, 2013; Lin et al., 2014), and therefore, may be potential therapies for neurodegenerative diseases including $\mathrm{AD}$ (Lee and Kim, 2017). We found p38 signaling was activated in $A D$ by $A \beta_{42}$ and down-regulated after MaR1 treatment, indicating that MaR1 may also play protective role via inhibiting p38 MAPK mediated inflammation.

The down-regulated PI3K/AKT and up-regulated p38 and ERK signaling in $\mathrm{AD}$ can induce caspase 3 expression, which is closely related to neuronal apoptosis and is considered as the terminal event preceding cell death. Caspase 3 levels have been demonstrated to be higher in $\mathrm{AD}$ brains than in age-matched controls (Shimohama et al., 1999), the same as we have observed in the present study. However, we found that caspase 3 was down-regulated after MaR1 treatment, suggesting that MaR1 plays a protective role by inhibiting apoptosis. To sum up, MaR1 promotes neuronal survival and ameliorates inflammation by up-regulating PI3K/AKT, ERK pathway while down-regulating mTOR, p38, and caspase 3 pathway.

There are some limitations in the current study that can be addressed in further investigations. Firstly, in this study, $\mathrm{AD}$ animal model used was made by injecting $\mathrm{A} \beta_{42}$ into the hippocampus. Although this method is simple and economical, it could not imitate the pathological changes of $\mathrm{AD}$ patients completely, and AD transgenic mice may be a better choice. Secondly, the pathways affected by MaR1 are not only limited to inflammatory pathways, but also other proteins that affect cell survival, axonal growth, and apoptosis, other proteins besides inflammatory cytokines should also be investigated. Thirdly, MaR1 was administered intraventricularly to avoid the restriction of blood-brain barrier (BBB). However, the capacity of MaR1 across the BBB needs to be further studied.

To conclude, resolution of inflammation is impaired in $\mathrm{AD}, \mathrm{MaR} 1$ could ameliorate memory dysfunction, improve neuronal survival, and reduce secretion of pro-inflammatory while promote anti-inflammatory mediators. MaR1 is beneficial in $\mathrm{AD}$ via pathways not only restricted to inflammation, but also through pathways involved in cellular survival, autophagy, axon formation, and apoptosis.

\section{DATA AVAILABILITY STATEMENT}

All datasets generated for this study are included in the manuscript/supplementary files.

\section{ETHICS STATEMENT}

All procedures used in the present study followed the "National Institutes of Health Guide for Care and Use of Laboratory Animals" (Publication No. 85-23, revised 1985) and were approved by the Animal Ethics Committee of Jilin University. Efforts were also made to minimize animal suffering and to reduce the number of animals used.

\section{AUTHOR CONTRIBUTIONS}

MZ, JF, PY, and XW designed the research. PY, XW, and YW performed the experiments. $M Z$ and $P Y$ analyzed the data. MZ, JF, PY, and XW wrote the manuscript. All authors reviewed the manuscript.

\section{FUNDING}

This study was supported by grants from the National Natural Science Foundation of China (Nos. 31600820 and 81701158), the Health and Family Planning Commission of Jilin Province (No. 2016Q036), the Science and Technology Planning Project of Jilin Province (No. 20180520110JH), the First Hospital of Jilin University (JDYY52015016) as well as from the Norman Bethune Program of Jilin University (No. 2015335).

\section{ACKNOWLEDGMENTS}

We would like to thank Editage for English language editing. 


\section{REFERENCES}

Alves, S., Churlaud, G., Audrain, M., Michaelsen-Preusse, K., Fol, R., Souchet, B., et al. (2017). Interleukin-2 improves amyloid pathology, synaptic failure and memory in Alzheimer's disease mice. Brain 140, 826-842. doi: 10.1093/brain/ aww330

Alzheimer, A., Stelzmann, R. A., Schnitzlein, H. N., and Murtagh, F. R. (1995). An english translation of Alzheimer's 1907 paper, "Uber eine eigenartige Erkankung der Hirnrinde”. Clin. Anat. 8, 429-431. doi: 10.1002/ca.980080612

Arora, K., Cheng, J., and Nichols, R. A. (2015). Nicotinic acetylcholine receptors sensitize a MAPK-linked toxicity pathway on prolonged exposure to betaamyloid. J. Biol. Chem. 290, 21409-21420. doi: 10.1074/jbc.M114.634162

Arthur, J. S., and Ley, S. C. (2013). Mitogen-activated protein kinases in innate immunity. Nat. Rev. Immunol. 13, 679-692. doi: 10.1038/nri3495

Bell, K. A., O’Riordan, K. J., Sweatt, J. D., and Dineley, K. T. (2004). MAPK recruitment by beta-amyloid in organotypic hippocampal slice cultures depends on physical state and exposure time. J. Neurochem. 91, 349-361. doi: 10.1111/j.1471-4159.2004.02722.x

Cacabelos, R., Alvarez, X. A., Fernandez-Novoa, L., Franco, A., Mangues, R., Pellicer, A., et al. (1994). Brain interleukin-1 $\beta$ in Alzheimer's disease and vascular dementia. Methods Find Exp. Clin. Pharmacol. 16, 141-151.

Chami, B., Steel, A. J., De La Monte, S. M., and Sutherland, G. T. (2016). The rise and fall of insulin signaling in Alzheimer's disease. Metab. Brain Dis. 31, 497-515. doi: 10.1007/s11011-016-9806-1

Chang, R., Yee, K. L., and Sumbria, R. K. (2017). Tumor necrosis factor alpha inhibition for Alzheimer's disease. J. Cent. Nerv. Syst. Dis. 9:1179573517709278. doi: $10.1177 / 1179573517709278$

Chong, Y. H., Shin, Y. J., Lee, E. O., Kayed, R., Glabe, C. G., and Tenner, A. J. (2006). ERK1/2 activation mediates Abeta oligomer-induced neurotoxicity via caspase3 activation and tau cleavage in rat organotypic hippocampal slice cultures. J. Biol. Chem. 281, 20315-20325. doi: 10.1074/jbc.M601016200

Cohen, S. B., Cheng, T. T., Chindalore, V., Damjanov, N., Burgos-Vargas, R., Delora, P., et al. (2009). Evaluation of the efficacy and safety of pamapimod, a p38 MAP kinase inhibitor, in a double-blind, methotrexate-controlled study of patients with active rheumatoid arthritis. Arthritis Rheum. 60, 335-344. doi: 10.1002/art.24266

Colaianna, M., Tucci, P., Zotti, M., Morgese, M. G., Schiavone, S., Govoni, S., et al. (2010). Soluble beta amyloid(1-42): a critical player in producing behavioural and biochemical changes evoking depressive-related state? $\mathrm{Br}$. J. Pharmacol. 159, 1704-1715. doi: 10.1111/j.1476-5381.2010.00669.x

Combs, C. K., Karlo, J. C., Kao, S. C., and Landreth, G. E. (2001). betaAmyloid stimulation of microglia and monocytes results in TNFalphadependent expression of inducible nitric oxide synthase and neuronal apoptosis. J. Neurosci. 21, 1179-1188. doi: 10.1523/jneurosci.21-04-01179.2001

Dalli, J., Vlasakov, I., Riley, I. R., Rodriguez, A. R., Spur, B. W., Petasis, N. A., et al. (2016). Maresin conjugates in tissue regeneration biosynthesis enzymes in human macrophages. Proc. Natl. Acad. Sci. U.S.A. 113, 12232-12237. doi: $10.1073 /$ pnas. 1607003113

Dalli, J., Zhu, M., Vlasenko, N. A., Deng, B., Haeggstrom, J. Z., Petasis, N. A., et al. (2013). The novel 13S,14S-epoxy-maresin is converted by human macrophages to maresin 1 (MaR1), inhibits leukotriene A4 hydrolase (LTA4H), and shifts macrophage phenotype. FASEB J. 27, 2573-2583. doi: 10.1096/fj.13-227728

Dansokho, C., Ait Ahmed, D., Aid, S., Toly-Ndour, C., Chaigneau, T., Calle, V., et al. (2016). Regulatory T cells delay disease progression in Alzheimer-like pathology. Brain 139(Pt 4), 1237-1251. doi: 10.1093/brain/awv408

Del Bo, R., Angeretti, N., Lucca, E., De Simoni, M. G., and Forloni, G. (1995). Reciprocal control of inflammatory cytokines, IL-1 and IL-6, and A $\beta$ production in cultures. Neurosci. Lett. 188, 70-74. doi: 10.1016/0304-3940(95) 11384-9

Deng, B., Wang, C. W., Arnardottir, H. H., Li, Y., Cheng, C. Y., Dalli, J., et al. (2014). Maresin biosynthesis and identification of maresin 2, a new anti-inflammatory and pro-resolving mediator from human macrophages. PLoS One 9:e102362. doi: 10.1371/journal.pone.0102362

Dineley, K. T., Westerman, M., Bui, D., Bell, K., Ashe, K. H., and Sweatt, J. D. (2001). Beta-amyloid activates the mitogen-activated protein kinase cascade via hippocampal alpha7 nicotinic acetylcholine receptors: in vitro and in vivo mechanisms related to Alzheimer's disease. J. Neurosci. 21, 4125-4133. doi: 10.1523/jneurosci.21-12-04125.2001
Dunn, H. C., Ager, R. R., Baglietto-Vargas, D., Cheng, D., Kitazawa, M., Cribbs, D. H., et al. (2015). Restoration of lipoxin A4 signaling reduces Alzheimer's disease-like pathology in the 3xTg-AD mouse model. J. Alzheimers Dis. 43, 893-903. doi: 10.3233/JAD-141335

Fredman, G., Hellmann, J., Proto, J. D., Kuriakose, G., Colas, R. A., Dorweiler, B., et al. (2016). An imbalance between specialized pro-resolving lipid mediators and pro-inflammatory leukotrienes promotes instability of atherosclerotic plaques. Nat. Commun. 7:12859. doi: 10.1038/ncomms12859

Gao, Y., Min, K., Zhang, Y., Su, J., Greenwood, M., and Gronert, K. (2015). Female-specific downregulation of tissue polymorphonuclear neutrophils drives impaired regulatory $\mathrm{T}$ cell and amplified effector $\mathrm{T}$ cell responses in autoimmune dry eye disease. J. Immunol. 195, 3086-3099. doi: 10.4049/ jimmunol.1500610

Garcia, J. M., Stillings, S. A., Leclerc, J. L., Phillips, H., Edwards, N. J., Robicsek, S. A., et al. (2017). Role of interleukin-10 in acute brain injuries. Front. Neurol. 8:244. doi: 10.3389/fneur.2017.00244

Gezen-Ak, D., Dursun, E., Hanagasi, H., Bilgic, B., Lohman, E., Araz, O. S., et al. (2013). BDNF, TNFalpha, HSP90, CFH, and IL-10 serum levels in patients with early or late onset Alzheimer's disease or mild cognitive impairment. J. Alzheimers Dis. 37, 185-195. doi: 10.3233/JAD-130497

Gilbert, K., Bernier, J., Bourque-Riel, V., Malick, M., and Rousseau, G. (2015). Resolvin D1 reduces infarct size through a phosphoinositide 3-kinase/protein kinase b mechanism. J. Cardiovasc. Pharmacol. 66, 72-79. doi: 10.1097/FJC. 0000000000000245

Griciuc, A., Serrano-Pozo, A., Parrado, A. R., Lesinski, A. N., Asselin, C. N., Mullin, K., et al. (2013). Alzheimer's disease risk gene CD33 inhibits microglial uptake of amyloid- $\beta$. Neuron 78, 631-643. doi: 10.1016/j.neuron.2013.04.014

Griffin, W. S., Stanley, L. C., Ling, C., White, L., MacLeod, V., Perrot, L. J., et al. (1989). Brain interleukin 1 and S-100 immunoreactivity are elevated in down syndrome and Alzheimer disease. Proc. Natl. Acad. Sci. U.S.A. 86, 7611-7615. doi: $10.1073 /$ pnas.86.19.7611

Guillot-Sestier, M. V., Doty, K. R., Gate, D., Rodriguez, J. Jr., Leung, B. P., RezaiZadeh, K., et al. (2015a). Il10 deficiency rebalances innate immunity to mitigate Alzheimer-like pathology. Neuron 85, 534-548. doi: 10.1016/j.neuron.2014. 12.068

Guillot-Sestier, M. V., Doty, K. R., and Town, T. (2015b). Innate immunity fights Alzheimer's disease. Trends Neurosci. 38, 674-681. doi: 10.1016/j.tins.2015. 08.008

Halapin, N. A., and Bazan, N. G. (2010). NPD1 induction of retinal pigment epithelial cell survival involves PI3K/Akt phosphorylation signaling. Neurochem. Res. 35, 1944-1947. doi: 10.1007/s11064-010-0351-358

Hao, Y., Zheng, H., Wang, R. H., Li, H., Yang, L. L., Bhandari, S., et al. (2019). Maresin1 alleviates metabolic dysfunction in septic mice: a (1)H NMR-based metabolomics analysis. Mediators Inflamm. 2019:2309175. doi: 10.1155/2019/ 2309175

Hardy, J. (2009). The amyloid hypothesis for Alzheimer's disease: a critical reappraisal. J. Neurochem. 110, 1129-1134. doi: 10.1111/j.1471-4159.2009. 06181.x

Hensley, K., Floyd, R. A., Zheng, N. Y., Nael, R., Robinson, K. A., Nguyen, X., et al. (1999). p38 kinase is activated in the Alzheimer's disease brain. J. Neurochem. 72, 2053-2058.

Heppner, F. L., Ransohoff, R. M., and Becher, B. (2015). Immune attack: the role of inflammation in Alzheimer disease. Nat. Rev. Neurosci. 16, 358-372. doi: $10.1038 / \mathrm{nrn} 3880$

Hickman, S. E., Allison, E. K., and El Khoury, J. (2008). Microglial dysfunction and defective beta-amyloid clearance pathways in aging Alzheimer's disease mice. J. Neurosci. 28, 8354-8360. doi: 10.1523/JNEUROSCI.0616-08.2008

Kang, J., and Rivest, S. (2012). Lipid metabolism and neuroinflammation in Alzheimer's disease: a role for liver X receptors. Endocr. Rev. 33, 715-746. doi: 10.1210/er.2011-1049

Kim, K. C., Lee, I. K., Kang, K. A., Cha, J. W., Cho, S. J., Na, S. Y., et al. (2013). 7,8-Dihydroxyflavone suppresses oxidative stress-induced base modification in DNA via induction of the repair enzyme 8-oxoguanine DNA glycosylase-1. Biomed. Res. Int. 2013:863720. doi: 10.1155/2013/863720

Kim, S., Jing, K., Shin, S., Jeong, S., Han, S. H., Oh, H., et al. (2018). omega3polyunsaturated fatty acids induce cell death through apoptosis and autophagy in glioblastoma cells: in vitro and in vivo. Oncol. Rep. 39, 239-246. doi: 10.3892/ or.2017.6101 
Kriplani, N., Hermida, M. A., Brown, E. R., and Leslie, N. R. (2015). Class I PI 3kinases: function and evolution. Adv. Biol. Regul. 59, 53-64. doi: 10.1016/j.jbior. 2015.05.002

Lee, J. K., and Kim, N. J. (2017). Recent advances in the inhibition of p38 MAPK as a potential strategy for the treatment of Alzheimer's disease. Molecules 22:E1287. doi: 10.3390/molecules22081287

Lin, X., Wang, M., Zhang, J., and Xu, R. (2014). p38 MAPK: a potential target of chronic pain. Curr. Med. Chem. 21, 4405-4418. doi: 10.2174/ 0929867321666140915143040

Lukiw, W. J., Cui, J. G., Marcheselli, V. L., Bodker, M., Botkjaer, A., Gotlinger, K., et al. (2005). A role for docosahexaenoic acid-derived neuroprotectin D1 in neural cell survival and Alzheimer disease. J. Clin. Invest. 115, 2774-2783. doi: 10.1172/JCI25420

Marber, M. S., Rose, B., and Wang, Y. (2011). The p38 mitogen-activated protein kinase pathway-a potential target for intervention in infarction, hypertrophy, and heart failure. J. Mol. Cell Cardiol. 51, 485-490. doi: 10.1016/j.yjmcc.2010. 10.021

McGeer, P. L., Itagaki, S., Boyes, B. E., and McGeer, E. G. (1988). Reactive microglia are positive for HLA-DR in the substantia nigra of Parkinson's and Alzheimer's disease brains. Neurology 38, 1285-1291.

McGeer, P. L., Itagaki, S., Tago, H., and McGeer, E. G. (1987). Reactive microglia in patients with senile dementia of the Alzheimer type are positive for the histocompatibility glycoprotein HLA-DR. Neurosci. Lett. 79, 195-200. doi: 10.1016/0304-3940(87)90696-3

Medeiros, R., Kitazawa, M., Passos, G. F., Baglietto-Vargas, D., Cheng, D., Cribbs, D. H., et al. (2013). Aspirin-triggered lipoxin A4 stimulates alternative activation of microglia and reduces Alzheimer disease-like pathology in mice. Am. J. Pathol. 182, 1780-1789. doi: 10.1016/j.ajpath.2013.01.051

Michaud, J. P., and Rivest, S. (2015). Anti-inflammatory signaling in microglia exacerbates Alzheimer's disease-related pathology. Neuron 85, 450-452. doi: 10.1016/j.neuron.2015.01.021

Murphy, G. M. Jr., Yang, L., and Cordell, B. (1998). Macrophage colony-stimulating factor augments beta-amyloid-induced interleukin-1, interleukin-6, and nitric oxide production by microglial cells. J. Biol. Chem. 273, 20967-20971. doi: $10.1074 /$ jbc.273.33.20967

O'Neill, C. (2013). PI3-kinase/Akt/mTOR signaling: impaired on/off switches in aging, cognitive decline and Alzheimer's disease. Exp. Gerontol. 48, 647-653. doi: 10.1016/j.exger.2013.02.025

Passos, G. F., Figueiredo, C. P., Prediger, R. D., Silva, K. A., Siqueira, J. M., Duarte, F. S., et al. (2010). Involvement of phosphoinositide 3-kinase gamma in the neuro-inflammatory response and cognitive impairments induced by beta-amyloid 1-40 peptide in mice. Brain Behav. Immun. 24, 493-501. doi: 10.1016/j.bbi.2009.12.003

Prieto, P., Cuenca, J., Traves, P. G., Fernandez-Velasco, M., Martin-Sanz, P., and Bosca, L. (2010). Lipoxin A4 impairment of apoptotic signaling in macrophages: implication of the PI3K/Akt and the ERK/Nrf-2 defense pathways. Cell Death Differ. 17, 1179-1188. doi: 10.1038/cdd.2009.220

Prokop, S., Miller, K. R., and Heppner, F. L. (2013). Microglia actions in Alzheimer's disease. Acta Neuropathol. 126, 461-477. doi: 10.1007/s00401-013-1182-x

Recabarren, D., and Alarcon, M. (2017). Gene networks in neurodegenerative disorders. Life Sci. 183, 83-97. doi: 10.1016/j.lfs.2017.06.009

Ringheim, G. E., Szczepanik, A. M., Petko, W., Burgher, K. L., Zhu, S. Z., and Chao, C. C. (1998). Enhancement of beta-amyloid precursor protein transcription and expression by the soluble interleukin- 6 receptor/interleukin- 6 complex. Brain Res. Mol. Brain Res. 55, 35-44. doi: 10.1016/s0169-328x(97)00356-2

Russo, C., Dolcini, V., Salis, S., Venezia, V., Zambrano, N., Russo, T., et al. (2002). Signal transduction through tyrosine-phosphorylated C-terminal fragments of amyloid precursor protein via an enhanced interaction with Shc/Grb2 adaptor proteins in reactive astrocytes of Alzheimer's disease brain. J. Biol. Chem. 277, 35282-35288. doi: 10.1074/jbc.M110785200

Saura, J., Tusell, J. M., and Serratosa, J. (2003). High-yield isolation of murine microglia by mild trypsinization. Glia 44, 183-189. doi: 10.1002/glia. 10274

Schnoder, L., Hao, W., Qin, Y., Liu, S., Tomic, I., Liu, X., et al. (2016). Deficiency of neuronal p38alpha MAPK attenuates amyloid pathology in alzheimer disease mouse and cell models through facilitating lysosomal degradation of BACE1. J. Biol. Chem. 291, 2067-2079. doi: 10.1074/jbc.M115.695916

Serhan, C. N. (2014). Pro-resolving lipid mediators are leads for resolution physiology. Nature 510, 92-101. doi: 10.1038/nature13479
Serhan, C. N. (2017). Treating inflammation and infection in the 21st century: new hints from decoding resolution mediators and mechanisms. FASEB J. 31, 1273-1288. doi: 10.1096/fj.201601222R

Shimohama, S., Tanino, H., and Fujimoto, S. (1999). Changes in caspase expression in Alzheimer's disease: comparison with development and aging. Biochem. Biophys. Res. Commun. 256, 381-384. doi: 10.1006/bbrc.1999.0344

Singh-Manoux, A., Dugravot, A., Brunner, E., Kumari, M., Shipley, M., Elbaz, A., et al. (2014). Interleukin-6 and C-reactive protein as predictors of cognitive decline in late midlife. Neurology 83, 486-493. doi: 10.1212/WNL. 0000000000000665

Strle, K., Zhou, J. H., Shen, W. H., Broussard, S. R., Johnson, R. W., Freund, G. G., et al. (2001). Interleukin-10 in the brain. Crit. Rev. Immunol. 21, 427-449.

Sun, A., Liu, M., Nguyen, X. V., and Bing, G. (2003). P38 MAP kinase is activated at early stages in Alzheimer's disease brain. Exp. Neurol. 183, 394-405. doi: 10.1016/s0014-4886(03)00180-8

Sun, Q., Wu, Y., Zhao, F., and Wang, J. (2017). Maresin 1 ameliorates lung ischemia/reperfusion injury by suppressing oxidative stress via activation of the Nrf-2-mediated HO-1 signaling pathway. Oxid. Med. Cell Longev. 2017:9634803. doi: 10.1155/2017/9634803

Tang, Q., Che, C., Lin, J., He, H., Zhao, W., Lv, L., et al. (2019). Maresin1 regulates neutrophil recruitment and IL-10 expression in Aspergillus Fumigatus keratitis. Int Immunopharmacol 69, 103-108. doi: 10.1016/j.intimp.2019.01.032

Tramutola, A., Lanzillotta, C., Barone, E., Arena, A., Zuliani, I., Mosca, L., et al. (2018). Intranasal rapamycin ameliorates Alzheimer-like cognitive decline in a mouse model of down syndrome. Transl. Neurodegener. 7:28. doi: 10.1186/ s40035-018-0133-9

Tramutola, A., Lanzillotta, C., and Di Domenico, F. (2017). Targeting mTOR to reduce Alzheimer-related cognitive decline: from current hits to future therapies. Expert Rev. Neurother. 17, 33-45. doi: 10.1080/14737175.2017. 1244482

Wang, X., Zhu, M., Hjorth, E., Cortes-Toro, V., Eyjolfsdottir, H., Graff, C., et al. (2015). Resolution of inflammation is altered in Alzheimer's disease. Alzheimers Dement. 11:40-50.e1-2. doi: 10.1016/j.jalz.2013.12.024

Wang, Y., Wu, C., Han, B., Xu, F., Mao, M., Guo, X., et al. (2016). Dexmedetomidine attenuates repeated propofol exposure-induced hippocampal apoptosis, PI3K/Akt/Gsk-3beta signaling disruption, and juvenile cognitive deficits in neonatal rats. Mol. Med. Rep. 14, 769-775. doi: 10.3892/mmr.2016.5321

Wicklund, L., Leao, R. N., Stromberg, A. M., Mousavi, M., Hovatta, O., Nordberg, A., et al. (2010). A $\beta 42$ oligomers impair function of human embryonic stem cell-derived forebrain cholinergic neurons. PLoS One 5:e15600. doi: 10.1371/ journal.pone.0015600

Yurko-Mauro, K., McCarthy, D., Rom, D., Nelson, E. B., Ryan, A. S., Blackwell, A., et al. (2010). Beneficial effects of docosahexaenoic acid on cognition in agerelated cognitive decline. Alzheimers Dement. 6, 456-464. doi: 10.1016/j.jalz. 2010.01.013

Zhang, J. L., Zhuo, X. J., Lin, J., Luo, L. C., Ying, W. Y., Xie, X., et al. (2017). Maresin1 stimulates alveolar fluid clearance through the alveolar epithelial sodium channel Na,K-ATPase via the ALX/PI3K/Nedd4-2 pathway. Lab. Invest. 97, 543-554. doi: 10.1038/labinvest.2016.150

Zhu, M., Wang, X., Hjorth, E., Colas, R. A., Schroeder, L., Granholm, A. C., et al. (2016). Pro-resolving lipid mediators improve neuronal survival and increase Abeta42 phagocytosis. Mol. Neurobiol. 53, 2733-2749. doi: 10.1007/s12035015-9544-0

Zilka, N., Kazmerova, Z., Jadhav, S., Neradil, P., Madari, A., Obetkova, D., et al. (2012). Who fans the flames of Alzheimer's disease brains? Misfolded tau on the crossroad of neurodegenerative and inflammatory pathways. J. Neuroinflamm. 9:47. doi: 10.1186/1742-2094-9-47

Conflict of Interest: The authors declare that the research was conducted in the absence of any commercial or financial relationships that could be construed as a potential conflict of interest.

Copyright (c) 2019 Yin, Wang, Wang, Wei, Feng and Zhu. This is an open-access article distributed under the terms of the Creative Commons Attribution License (CC BY). The use, distribution or reproduction in other forums is permitted, provided the original author(s) and the copyright owner(s) are credited and that the original publication in this journal is cited, in accordance with accepted academic practice. No use, distribution or reproduction is permitted which does not comply with these terms. 